\title{
Cooperative interactions within a marine bacterial dual species biofilm growing on a natural biodegradable substratum
}

\author{
Kim J. Everuss*, Marina W. Delpin, Amanda E. Goodman \\ School of Biological Sciences, Flinders University, GPO BOX 2100, Adelaide, South Australia 5001, Australia
}

\begin{abstract}
Pseudoalteromonas sp. S91 is a marine bacterium known to secrete chitinases and proteases, hydrolytic enzymes responsible for the degradation of chitin and protein, respectively, which enable access to nutrients contained in chitinous materials such as squid pen. In a dual species biofilm grown on squid pen, Pseudoalteromonas sp. S91 was able to support the accumulation of Vibrio sp. S141, which is unable to degrade squid pen but able to metabolise the chitin subunit $N^{\prime}$-acetylglucosamine (GlcNAc), a product of squid pen hydrolysis. When grown on a glass substratum in the presence of a soluble carbon source that only Pseudoalteromonas sp. S91 could use, its biofilm provided no support to Vibrio sp. S141.
\end{abstract}

KEY WORDS: Chitin $\cdot$ Biofilm $\cdot$ Marine bacteria $\cdot$ Commensalism

\section{INTRODUCTION}

Bacterial populations within marine environments predominately exist as biofilms adhering to surfaces (Costerton et al. 1995). Within the dynamic biofilm environment, interspecies interactions directly influence biofilm morphology and species success (Nielsen et al. 2000, Christensen et al. 2002). Møller et al. (1997) found that nutrient concentration directly affects development of multispecies biofilms by influencing the location of species within the biofilm, while Rao et al. (2005) suggested that the formation of microcolonies by different species enhances their ability to persist under competitive conditions in mixed-species biofilms. Throughout the marine environment, biofilms predominately occur on biodegradable substrata (Costerton et al. 1994), such as marine snow, and usually consist of multiple species; analysis of species behaviour under such conditions has been little studied to date.

Past and current microbial research has concentrated on investigating the characteristics of pure species cultures supplemented with soluble carbon sources (Davies et al. 1998, O'Toole \& Kolter 1998,
Pratt \& Kolter 1998, Sternberg et al. 1999). Studies of mixed-species biofilms have predominately used inert surfaces (Wolfaardt et al. 1994, Palmer et al. 2001, Rao et al. 2005). However, bacteria within natural environments exist in mixed-species biofilms exposed to heterogeneous sources of nutrients and nutrient-rich surfaces. In studies that have analysed biodegradable compounds exposed to single- or mixed-species biofilm consortia, data collected have principally reported the rate of substrate degradation, and how this correlates with numbers within a bacterial consortium (Kudo et al. 1987, O'Sullivan et al. 2005, Song et al. 2005).

Recent studies investigating multispecies microbial consortia have focused on bacterial interactions within biofilms degrading compounds of environmental or commercial significance (Møller et al. 1997, De Souza et al. 1998, Nielsen et al. 2000). This research commonly views bacteria as immobilised enzymes able to hydrolyse a specific substrate with efficiency dependent upon the identity of the species present in the biofilm. Increases in degradation rate of the specific substrate by the biofilm can often be related to the presence in the biofilm of a second species, which 
while unable to directly degrade the specific substrate itself, instead degrades a toxic or inhibitory metabolic by-product of the first species (De Souza et al. 1998). Although microbial metabolism of dissolved organic carbon sources has also been subject to extensive study, the investigation of microbial metabolism of particulate organic matter (POM) is much less common (Vetter \& Deming 1999). It has been found that the mode of bacterial attachment, biofilm architecture and rate of biofilm development may be altered in response to the type of substratum it is grown upon (Dalton et al. 1994, Baty et al. 2000, Delpin et al. 2000). To better understand the interactions between different bacterial species within mixed-species biofilms when degrading the substratum to which they are attached, we used a model marine system, which combines a Pseudoalteromonas sp. with either a Vibrio sp. or a Psychrobacter sp. forming biofilms on a natural biodegradable substratum, squid pen, which is composed of $40 \%$ chitin and $60 \%$ protein (Gooday 1990). Chitin is composed of $\beta$-1-4-linked $N^{\prime}$-acetylglucosamine (GlcNAc) subunits, to which it can be hydrolysed by chitinases (Keyhani \& Roseman 1999); in squid pen, chitin has a $\beta$-crystalline structure (Gooday 1990). Chitin, a major component of oceanic $\mathrm{POM}$, is among the most abundant organic carbon and nitrogen sources in the marine environment, with approximately $10^{11} \mathrm{t}$ produced each year (Gooday 1990). The degradation of chitin and chitinous materials is predominately carried out by chitinase-producing micro-organisms (Gooday 1990), which are ubiquitous in the marine environment (Keyhani \& Roseman 1999).

The strains chosen for this study represent commonly culturable Gram-negative marine bacteria that can be genetically manipulated. Pseudoalteromonas sp. S91, a Gram-negative motile marine bacterium, is capable of recycling nutrients and energy contained in organic macromolecules and polymers via the production and subsequent action of hydrolytic enzymes, such as chitinases and proteases (Albertson et al. 1996). Squid pen can support Pseudoalteromonas sp. S91 growth as the sole carbon source (Techkarnjanaruk et al. 1997). Vibrio sp. S141, a Gram-negative motile marine bacterium (Albertson et al. 1996), also produces chitinases and proteases. Vibrio sp. S141 can grow on solid, non-crystalline chitin (Angles 1997), but in contrast to Pseudoalteromonas sp. S91, grows poorly with squid pen as the sole source of carbon. Psychrobacter sp. SW5H is a Gram-negative non-motile marine bacterium (Angles et al. 1993, Poulsen et al. 1997), which grows well at laboratory temperatures of 20 to $30^{\circ} \mathrm{C}$, and its affiliation to the genus Psychrobacter is based on 16srRNA gene sequencing (Poulsen et al. 1997). This strain does not produce chitinases or proteases and cannot grow on squid pen, chitin or GlcNAc as sole sources of carbon. Vibrio sp. S141 and Psychrobacter sp. SW5H, although unable to metabolise squid pen, are both capable of biofilm formation and plasmid transfer on the squid pen surface in the presence of a suitable soluble nutrient source (Angles 1997). A chitinase-negative mutant Pseudoalteromonas sp. S91CGFP was also used to investigate any role that chitinases may have during the interactions between Pseudoalteromonas sp. S91 and other species in dual-species biofilms on squid pen.

We analysed the interactions between different species in dual-species biofilms growing on squid pen as a biodegradable substratum, which acted as the sole source of carbon. To study dual-species biofilms, green fluorescent protein (GFP) was employed as a speciesspecific marker. Tagging of 1 strain enabled the spatial analysis of that strain within the biofilm by 2-photon laser scanning microscopy (TPLSM), and facilitated the real time qualitative and quantitative analysis of living cells within hydrated biofilms (Yoshida \& Kuramitsu 2002). Following specific inoculation regimes, one of the species was localised and quantified.

We found that the marine bacterium Pseudoalteromonas sp. S91 was able to support the accumulation of Vibrio sp. S141 when grown in dual-species biofilms on squid pen in the absence of a soluble carbon source. This relationship was altered when the capability of Pseudoalteromonas sp. S91 to produce chitinases was eliminated and when the biodegradable substratum was replaced with an inert substratum. In contrast, we found that Pseudoalteromonas sp. S91 was unable to provide similar support to Psychrobacter sp. SW5H when grown in dual-species biofilms on squid pen.

\section{MATERIALS AND METHODS}

Bacterial strains and plasmids. The strains and plasmids used in this study are listed in Table 1. Pseudoalteromonas sp. Strain S91SB is a derivative of Strain S91 (Techkarnjanaruk et al. 1997) into which a gfp gene has been inserted. Pseudoalteromonas sp. Strain S91CGFP, also a derivative of Strain S91, was constructed by transposon mutagenesis of the chiA gene with a promoterless transposon cassette containing a gfp gene (Matthysse et al. 1996). The plasmid p519ngfp is derived from the broad host range IncQRSF1010 derivative PDSK519 (Keen et al. 1988) and has an $n p t 2$ promoter constitutively expressing the $g f p$ gene (Matthysse et al. 1996).

Growth conditions. Marine bacteria were grown in batch at $30^{\circ} \mathrm{C}$ in either tryptone soy broth (TSB; Oxoid) containing $\mathrm{NaCl}(0.26 \mathrm{M}), \mathrm{MgCl}_{2}(1 \mathrm{mM})$ and $\mathrm{CaCl}_{2}$ 
Table 1. Strains and plasmids used in the study. GFP: green fluorescent protein; $\mathrm{Sm}^{\mathrm{R}}$ : streptomycin resistant; Km ${ }^{\mathrm{R}}$ : kanamycin resistant; + : positive ; $_{- \text {: negative }}$

\begin{tabular}{|c|c|c|c|c|}
\hline Bacterial strain or plasmid & GFP & Chitinase & Characteristic(s) & Source \\
\hline \multicolumn{5}{|l|}{ Bacterial strains } \\
\hline Pseudoalteromonas sp. & - & + & & \\
\hline Strain S91 & - & + & $\mathrm{Sm}^{\mathrm{R}}$ & Albertson et al. (1996) \\
\hline Strain S91CX & - & - & $\mathrm{Sm}^{\mathrm{R}}, \mathrm{Km}^{\mathrm{R}}$ & Techkarnjanaruk et al. (1997) \\
\hline Strain S91CGFP & + & - & $\mathrm{Sm}^{\mathrm{R}}, \mathrm{Km}^{\mathrm{R}}$ & Stretton et al. (1998) \\
\hline Strain S91SB & + & + & $\mathrm{Sm}^{\mathrm{R}}, \mathrm{Km}^{\mathrm{R}}$ & E. M. Schaffer (unpubl.) \\
\hline Vibrio sp. Strain S141 & - & + & $\mathrm{Sm}^{\mathrm{R}}$ & Östling et al. (1991) \\
\hline Psychrobacter sp. & - & - & $\mathrm{Sm}^{\mathrm{R}}$ & Angles et al. (1993) \\
\hline Strain SW5\#19 & - & - & $\mathrm{Sm}^{\mathrm{R}} \mathrm{Km}^{\mathrm{R}}$ & This study \\
\hline Escherichia coli SM10 & - & - & $\mathrm{Km}^{\mathrm{R}}$ & Miller \& Mekalanos (1988) \\
\hline \multicolumn{5}{|l|}{ Plasmids } \\
\hline p519gfp & + & & $\mathrm{Km}^{\mathrm{R}} \mathrm{mob}^{+}$ & Matthysse et al. (1996) \\
\hline p519ngfp & + & & $n p t-2$ promoter in front of $g f p$ & Matthysse et al. (1996) \\
\hline pDSK519 & - & & $\mathrm{Km}^{\mathrm{R}} \mathrm{mob}^{+}$, nonconjunjugative & Keen et al. (1988) \\
\hline pLOFKmgfp & + & & Promoterless $g f p, \mathrm{Km}^{\mathrm{R}}$ & Stretton et al. (1998) \\
\hline
\end{tabular}

(0.33 mM); Luria broth (LB) (Miller 1972) containing $\mathrm{NaCl}(0.26 \mathrm{M}), \mathrm{MgCl}_{2}(1 \mathrm{mM})$ and $\mathrm{CaCl}_{2}(0.33 \mathrm{mM})$; or artificial seawater minimal medium (Östling et al. 1991) supplemented with $20 \mathrm{mM}$ glutamate (MMM $\mathrm{glt}_{20}$ ) for Psychrobacter sp. SW5H strains, $40 \mathrm{mM}$ glutamate $\left(\mathrm{MMM} \mathrm{glt}_{40}\right)$ for Pseudoalteromonas sp. S91 strains or $20 \mathrm{mM}$ glucose $\left(\mathrm{MMM}_{\mathrm{gluc}} \mathrm{C}_{20}\right.$ ) for Vibrio sp. S141 strains. Pseudoalteromonas sp. S91 and Psychrobacter sp. SW5H strains are able to use glutamate, but not glucose, as a sole carbon source, whereas Vibrio sp. S141 can use glucose but not glutamate. Escherichia coli was grown in batch at $37^{\circ} \mathrm{C}$ in LB supplemented with $5 \mathrm{gl}^{-1} \mathrm{NaCl}$ (LB5). Kanamycin (Km) and streptomycin $(\mathrm{Sm})$ were both used at $100 \mu \mathrm{g} \mathrm{ml}^{-1}$; $\mathrm{Km}$ was included for plasmid maintenance where appropriate.

Transposon mutagenesis and isolation of strain Psychrobacter sp. SW5\#19. The vector pLOFKmgfp carrying a mini-Tn-10:gfp:kan transposon (Stretton et al. 1998) was transferred from the donor strain Escherichia coli SM10 to the recipient (Psychrobacter sp. SW5H) using the filter mating protocol described by Albertson et al. (1996). From log phase cultures (OD595 approximately 0.35) of donor and recipient cells, $50 \mu$ l were gently mixed and spotted on a sterile $0.2 \mu \mathrm{m}$ Millipore membrane filter placed on an LB plate supplemented with $15 \mathrm{~g} \mathrm{l}^{-1} \mathrm{NaCl}$ (LB15). Controls of each strain were spotted on separate filters on individual plates. After overnight inoculation, filters were separately resuspended in $1 \mathrm{ml} \mathrm{LB15}$ and then spread onto LB15 Sm Km plates to enable counter selection of donors and recipients. Seventy transconjugants, selected at appropriate dilutions on LB15 Sm $\mathrm{Km}$ plates, were screened for GFP fluorescence as colonies on the same medium (Stretton et al. 1998). Twenty-five positive strains were purified on MMM glt $_{20} \mathrm{Sm} \mathrm{Km}$ plates; wet mounts of each were screened for constitutive GFP production by epifluorescence microscopy. Six positive strains were grown in liquid MMM glt ${ }_{20} \mathrm{Sm} \mathrm{Km}$, and their growth rates were compared to Psychrobacter sp. SW5H. Transconjugant S Psychrobacter sp. SW5\#19 was selected for further use, as its growth rate was similar to that of Psychrobacter sp. SW5H (data not shown).

Squid pen. Squid pen pieces (Stretton et al. 1998) were cut into approximately $100 \mathrm{~mm}^{2}$ pieces (between the ridges) for use in experiments.

Squid pen growth test. Pseudoalteromonas sp. S91SB, Vibrio sp. S141(p519ngfp) and Psychrobacter sp. SW5\#19 were inoculated into flasks, each containing a single piece of squid pen and $10 \mathrm{ml}$ of MMM with no carbon supplement. Shake flasks were incubated at $30^{\circ} \mathrm{C}$ for up to $15 \mathrm{wk}$. By $15 \mathrm{wk}$ Pseudoalteromonas sp. S91SB produced thick visible biofilm on the pen within a few days and had completely degraded the pen piece, whereas the chitinase-negative mutant, Pseudoalteromonas sp. S91CX, did not fully degrade the pen, although it produced visible thick biofilm on it. In contrast, the Vibrio and Psychrobacter strains neither produced any visible biofilm on the pen nor planktonic growth, and squid pen pieces showed no degradation after $15 \mathrm{wk}$ of incubation.

Flow chamber experiments. Experiments were carried out using flow chambers based on the design of Hale \& Mitchell (2001) as used by Delpin et al. (2000) and Fitch et al. (2002). A rubber o-ring placed between 2 glass cover slips sealed the chamber and provided a viewing port. Two needles perforated the o-ring; one 
served as the inlet and the other as the outlet channel. The chamber volume was approximately $1 \mathrm{ml}$. At approximately 10 and $15 \mathrm{~cm}$ upstream from the chamber, 2 T-junctions were created in the silicone tubing, and these provided capped, sterile injection ports. Each injection port was used for the inoculation of 1 strain. Prior to assembly, an autoclaved sterile piece of squid pen was placed in each chamber. Assembled flow chambers and all tubing were sterilised by autoclaving and cleaned by pumping $70 \%$ ethanol through them for $15 \mathrm{~min}$ followed by sterile MMM for $20 \mathrm{~min}$. An 8-roller Cole-Palmer Masterflex peristaltic pump (7521-57) was used to pump sterile MMM through the chamber, at a flow rate of $1 \mathrm{ml} \mathrm{min}^{-1}$ (Delpin et al. 2000); the outlet of the chamber went to waste. Flow chambers containing squid pen were inoculated with $3 \mathrm{ml}$ of a $10^{-1}$ diluted overnight culture, grown with the appropriate carbon source at $30^{\circ} \mathrm{C}$ on a gyratory shaker. Where more than 1 species was inoculated at the same time, $1.5 \mathrm{ml}$ of a $10^{-1}$ dilution of each overnight culture was injected into the chamber at the same time through different injection ports. Chambers were left for $1 \mathrm{~h}$ without flow to allow cells to attach to the squid pen. After $1 \mathrm{~h}$, the flow was started and

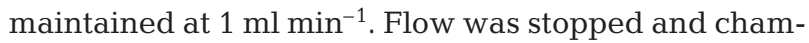
bers were disconnected after $72 \mathrm{~h}$, unless stated otherwise, and examined using a TPLSM.

To determine whether time or order of inoculation would affect the success of strains, 3 inoculation regimes were tested and are labelled as follows: $0 \mathrm{~h}$ means that the strain was inoculated at time $0\left(T_{0}\right)$, and allowed to develop a biofilm for $72 \mathrm{~h} ; 24 \mathrm{~h}$ means that the strain was inoculated $24 \mathrm{~h}$ after $T_{0}$ and allowed to develop for a further $48 \mathrm{~h}$. Each experiment was run for a total of $72 \mathrm{~h}$.

TPLSM. Following completion of flow chamber experiments, images of the bacterial biofilms within the chambers were collected using a Bio-Rad Radiance 2000MP visualising system in conjunction with a Nikon Eclipse TE300 inverted microscope. The microscope was equipped with a $60 \times$ water immersion lens with a numerical aperture of 1.2 and a Coherent Mira900-F titanium:sapphire ultra fast laser, which has an excitation spectrum of pulsed $800 \mathrm{~nm}$ light equivalent to 1 photon of $400 \mathrm{~nm}$ light, suitable for exciting GFP without damaging cells (Lim et al. 2007). An excitation wavelength $(\lambda)$ of 2 photons of $800 \mathrm{~nm}$ and an emission $\lambda$ of 515 to $530 \mathrm{~nm}$ was used to visualise GFP. An emission $\lambda$ of 450 to $480 \mathrm{~nm}$ was used to visualise squid pen autofluorescence. Each experiment was repeated at least once, so that a minimum of 2 flow chambers were analysed for each experiment. Images of the bacterial biofilms were collected as highest resolution horizontal $(x y)$ and vertical $(x z)$ optical sections and recorded as digital computer files. For each chamber, $9 \mathrm{xz}$ images were randomly captured and recorded. Images were converted using Confocal Assistant 4.02 (Todd Clarke Brelje, University of Minnesota). Image analysis was performed using ImageJ 1.32j (Abramoff et al. 2004) computer image analysis software.

Psychrobacter sp. SW5\#19, Pseudoalteromonas sp. S91SB and Vibrio sp. S141(p519ngfp) grown in batch monoculture were each viewed as wet mounts using the TPLSM. Horizontal xy sections of Psychrobacter sp. SW5\#19, Pseudoalteromonas sp. S91SB and Vibrio sp. S141(p519ngfp) biofilm cells were collected in the emission spectra $\lambda 515$ to $530 \mathrm{~nm}$. The mean area covered by a single $\mathrm{GFP}^{+}$cell was determined by measuring the collective area of 50 random cells. The mean area was $0.6,0.56$ and $1.86 \mu^{2}$ for a single cell of Psychrobacter sp. SW5\#19, Pseudoalteromonas sp. S91SB and Vibrio sp. S141(p519ngfp), respectively. Numbers of $\mathrm{GFP}^{+}$cells within $x z$ images in the green spectra were calculated from the total area showing fluorescence using ImageJ 1.32j (Abramoff et al. 2004). Images used for analysis with ImageJ 1.32j (Abramoff et al. 2004) were computer manipulated; a median filter of 2.0 was applied to all images to reduce noise, and the histogram was modified to exclude background pixels, focusing on bacteria. Presented TPLSM images were manipulated using Adobe Photoshop. The histogram was optimised and a colour hue applied separately to squid pen and GFP-positive cells where appropriate. For $x z$ sections, the negative image was falsely coloured.

Statistical analysis. To compare numbers of GFPtagged cells in biofilms between experiments, Student's $t$-test assuming unequal variances was used to compare means from each experiment. Formulae used were as described by Zar (1996). A standardised area of $x z$ sections was used when quantifying cell numbers: $96 \mu \mathrm{m}$ in length $(x)$ and $52 \mu \mathrm{m}$ in height $(z)$. For each experiment, 18 or more images, collected from chambers run in duplicate, were used to calculate mean cell numbers. Tables 2 to 4 show the numbers of GFP-tagged cells present in biofilms from each experiment.

\section{RESULTS}

Monoculture biofilms were grown on squid pen for 72 h. Pseudoalteromonas sp. S91 and derivative strains heavily colonised the pen and formed thick biofilms (Fig. 1a,b, Table 2). Vibrio sp. S141(p519ngfp) and Psychrobacter sp. SW5\#19 strains showed patchy colonisation of the pen, with single cells and microcolonies situated directly on the pen surface (Fig. 1c,d, Table 2). 


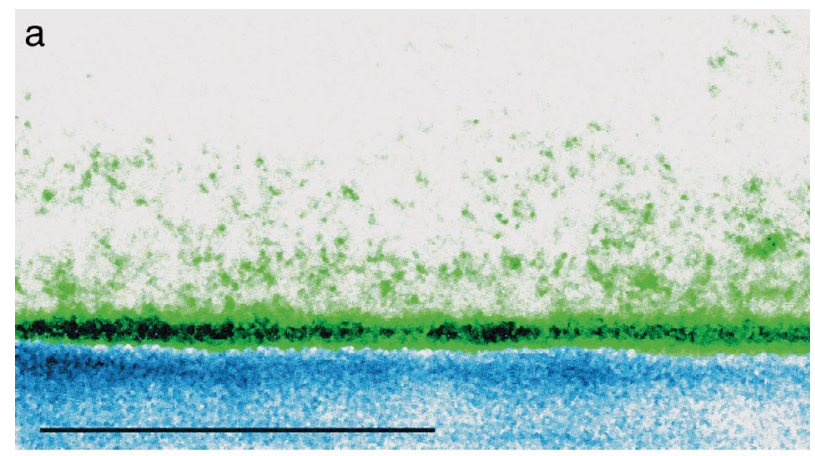

b

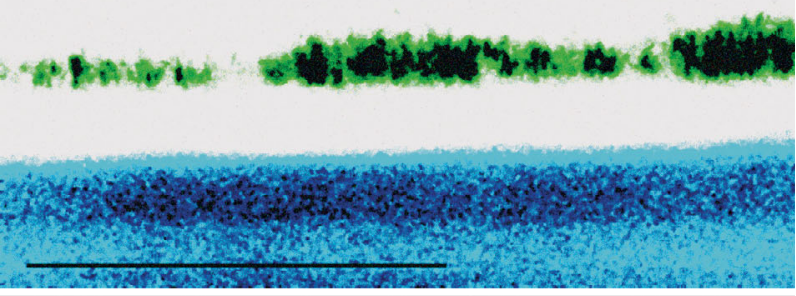

C

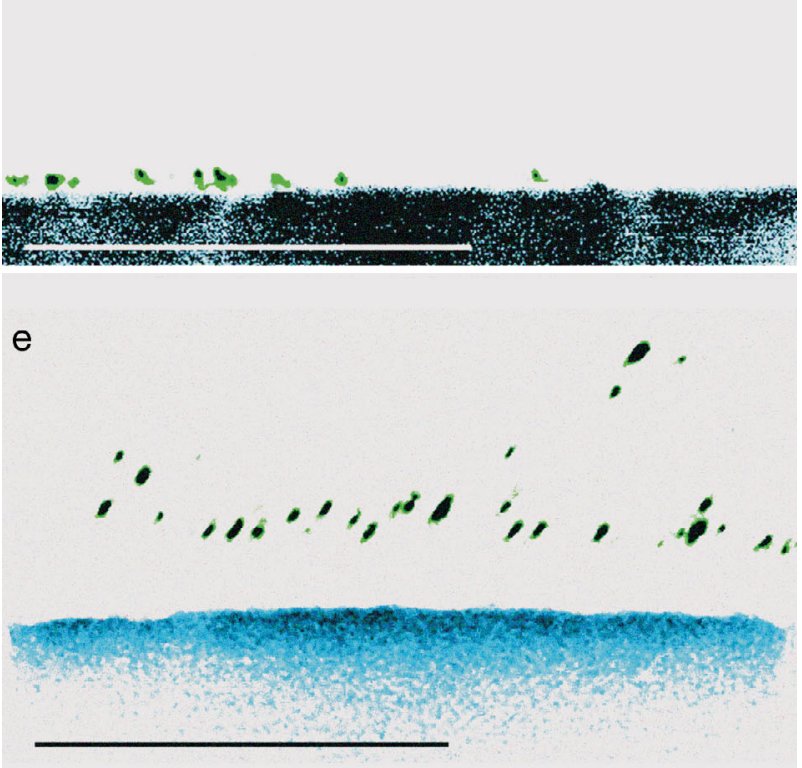

e

d

\section{Pseudoalteromonas sp. S91(pDSK519) and Psychrobacter sp. SW5\#19 interactions}

When Pseudoalteromonas sp. S91(pDSK519) colonised the pen first or both strains were inoculated together, squid pen pieces were completely colonised by Pseudoalteromonas sp. S91(pDSK519) only, with no Psychrobacter sp. SW5\#19 cells detected; when Psychrobacter sp. SW5\#19 cells were inoculated first, numbers detected after the addition of Pseudoalteromonas sp. S91(pDSK519) were significantly lower $(\mathrm{p}<0.05)$ than when Pseudoalteromonas sp. S91(pDSK519) was absent (Table 2). These data showed that the Psychrobacter SW5\#19 strain was not supported, and was in fact outcompeted by, Pseudoalteromonas sp. S91(pDSK519) on squid pen.

\section{Pseudoalteromonas sp. S91(pDSK519) and Vibrio sp. S141(p519ngfp) interactions}

Biofilm numbers of Vibrio sp. S141(p519ngfp) were significantly higher $(p<0.05)$ in the presence of Pseudoalteromonas sp. S91(pDSK519) than when grown in monoculture, independent of inoculation order (Table 2). Following all 3 inoculation regimes, squid pen pieces were heavily colonised by Pseudoalteromonas sp. S91(pDSK519) cells (seen by light microscopy, data not shown) which were always positioned between the Vibrio sp. S141(p519ngfp) cells and the pen (Fig. 1e).

Following the addition of either glucose or GlcNAc, the monomer subunit of chitin, for the first $24 \mathrm{~h}$ of a 72 h experiment, Vibrio sp. S141(p519ngfp) monoculture numbers were significantly greater $(p<0.05)$ than when no soluble carbon source was added for the same time period (Table 2). This experiment was repeated except that at $24 \mathrm{~h}$, when the feed of the soluble carbon source was discontinued, Pseudoalteromonas sp. S91(pDSK519) was inoculated. The numbers of Vibrio sp. S141(p519ngfp) under these conditions were significantly higher $(\mathrm{p}<0.05)$ than those when Vibrio sp. S141(p519ngfp) was grown in monoculture or dualspecies biofilms without the addition of a soluble carbon source for the first $24 \mathrm{~h}$ (Table 2). The results also showed that an increased accumulation of Vibrio sp.

Fig. 1. Artificially coloured $x z$ profiles of representative areas of green fluorescence protein (GFP) ${ }^{+}$biofilm cells (green) on squid pen (blue) after $72 \mathrm{~h}$. (a) GFP ${ }^{+}$Pseudoalteromonas sp. S91SB in monoculture; (b) $\mathrm{GFP}^{+}$Pseudoalteromonas sp. S91CGFP in monoculture; (c) GFP ${ }^{+}$Vibrio sp. S141(p519ngfp) in monoculture; (d) GFP ${ }^{+}$Psychrobacter sp. SW5\#19 in monoculture; (e) GFP ${ }^{+}$Vibrio sp. S141(p519ngfp) and Pseudoalteromonas sp. S91(pDSK519) mixed species. Scale bars $=50 \mu \mathrm{m}$ 
Table 2. Experimental inoculation regimes and numbers of $\mathrm{GFP}^{+}$cells after $72 \mathrm{~h}$ (mean $\pm \mathrm{SD}$ ) in biofilms on squid pen

\begin{tabular}{|c|c|c|}
\hline $\begin{array}{l}\text { Strain inoculation at } \\
\text { time } 0 \mathrm{~h}\end{array}$ & $\begin{array}{l}\text { Strain and time } \\
\text { of inoculation (h) }\end{array}$ & $\begin{array}{c}\mathrm{GFP}^{+} \text {cell numbers } \\
\text { per } 5000 \mu \mathrm{m}^{2} \text { after } 72 \mathrm{~h}\end{array}$ \\
\hline Pseudoalteromonas sp. S91SB & - & $1.5 \times 10^{3}\left( \pm 5.7 \times 10^{2}\right)$ \\
\hline Vibrio sp. S141(p519ngfp) & - & $2.2 \times 10^{0}\left( \pm 1.7 \times 10^{0}\right)$ \\
\hline Pseudoalteromonas sp. S91CGFP & - & $5.9 \times 10^{2}\left( \pm 1.3 \times 10^{2}\right)$ \\
\hline Pseudoalteromonas sp. S91(pDSK519) & Vibrio sp. S141(p519ngfp) (24 h) & $2.5 \times 10^{1}\left( \pm 1.4 \times 10^{1}\right)$ \\
\hline Vibrio sp. S141(p519ngfp) & Pseudoalteromonas sp. S91(pDSK519) (24 h) & $1.8 \times 10^{1}\left( \pm 0.7 \times 10^{1}\right)$ \\
\hline Vibrio sp. S141(p519ngfp) & Pseudoalteromonas sp. S91(pDSK519) (0 h) & $2.9 \times 10^{1}\left( \pm 2.1 \times 10^{1}\right)$ \\
\hline Vibrio sp. S141(p519ngfp) (Gluc for $24 \mathrm{~h}$ ) & - & $1.6 \times 10^{1}\left( \pm 1.1 \times 10^{1}\right)$ \\
\hline Vibrio sp. S141(p519ngfp) (Gluc for $24 \mathrm{~h}$ ) & Pseudoalteromonas sp. S91(pDSK519) (24 h) & $6.3 \times 10^{1}\left( \pm 4.7 \times 10^{1}\right)$ \\
\hline Vibrio sp. S141(p519ngfp) (GlcNac for $24 \mathrm{~h}$ ) & 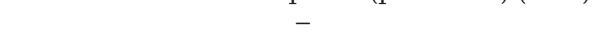 & $2.3 \times 10^{1}\left( \pm 1.3 \times 10^{1}\right)$ \\
\hline Vibrio sp. S141(p519ngfp) (GlcNac for $24 \mathrm{~h}$ ) & Pseudoalteromonas sp. S91(pDSK519) (24 h) & $2.3 \times 10^{1}\left( \pm 1.3 \times 10^{1}\right)$ \\
\hline Vibrio sp. S141(p519ngfp) (GlcNac for $24 \mathrm{~h}$ ) & Pseudoalteromonas sp. S91(pDSK519) (24 h) & $7.1 \times 10^{1}\left( \pm 3.1 \times 10^{1}\right)$ \\
\hline Psychrobacter sp. SW5\#19 & - & $7.7 \times 10^{1}\left( \pm 4.1 \times 10^{1}\right)$ \\
\hline Psychrobacter sp. SW5\#19 & Pseudoalteromonas sp. S91(pDSK519) (24 h) & $1.0 \times 10^{1}\left( \pm 0.8 \times 10^{1}\right)$ \\
\hline Pseudoalteromonas sp. S91SB & Vibrio sp. S141wt (0 h) & $1.6 \times 10^{3}\left( \pm 4.0 \times 10^{2}\right)$ \\
\hline
\end{tabular}

S141(p519ngfp) was supported during dual-species biofilm development on squid pen, since cell numbers were again significantly increased $(p<0.05)$ by the presence of Pseudoalteromonas sp. S91(pDSK519) (Table 2). In both mono- and dual-species biofilms, Vibrio sp. S141(p519ngfp) numbers were similar when either glucose or GlcNAc was used as the soluble carbon source for the initial $24 \mathrm{~h}$.

Vibrio sp. S141(p519ngfp) did not appear to affect the growth of Pseudoalteromonas sp. S91SB as its numbers were similar $(\mathrm{p}<0.05)$ in both mono- and dual-species biofilms (Table 2).

\section{Do the major Pseudoalteromonas sp. S91 chitinases play a role in supporting Vibrio sp. S141?}

When grown in the presence of the chitinase negative strain (Pseudoalteromonas sp. S91CX), the numbers of Vibrio sp. S141(p519ngfp) were significantly lower ( $p<0.05$; Table 3 ) following all 3 inoculation regimes than those grown in the presence of Pseudoalteromonas sp. S91(pDSK519) (Table 2) and similar to numbers grown in monoculture (Table 2). These data suggested that accumulation of the Vibrio sp. S141(p519ngfp) on squid pen was dependent on the chitinase-degrading ability of the Pseudoalteromonas sp. S91; therefore, the effect of biofilm growth on an abiotic surface was investigated.

\section{Effect of substratum on Vibrio sp. S141(p519ngfp)-Pseudoalteromonas sp. S91 interactions in dual-species biofilms}

To investigate the effect of the substratum on dualspecies biofilm development and species behaviour, the 2 species were grown on glass in the presence of glutamate, which Vibrio sp. S141(p519ngfp) is unable to use as a carbon source. After $72 \mathrm{~h}$, glass cover slips were heavily colonised by either Pseudoalteromonas sp. S91(pDSK519) or Pseudoalteromonas sp. S91CX, with Vibrio sp. S141(p519ngfp) cells predominately localised at the outer edge of the biofilms (data not shown). Vibrio sp. S141(p519ngfp) numbers were similar to those attached to the glass in monoculture biofilms in the presence of glutamate (Table 4). There was no significant difference in numbers when cells were grown with either Pseudoalteromonas sp. S91(pDSK519) or Pseudoalteromonas sp. S91CX, indicating that Vibrio sp. S141(p519ngfp) was able to coexist with, but was not supported by, Pseudoalteromonas sp. S91 biofilms growing on an inert substratum.

Table 3. Experimental inoculation regimes and numbers of $\mathrm{GFP}^{+}$cells (mean $\pm \mathrm{SD}$ ) in biofilms on squid pen

\begin{tabular}{|lcr|}
\hline Strain inoculation at time $0 \mathrm{~h}$ & Strain and time of inoculation (h) & $\begin{array}{c}\mathrm{GFP}^{+} \text {cell numbers } \\
\text { per } 5000 \mu \mathrm{m}^{2} \mathrm{after} 72 \mathrm{~h}\end{array}$ \\
\hline Pseudoalteromonas sp. S91CX & Vibrio sp. S141(p519ngfp) (24) & $1.7 \times 10^{0}\left( \pm 1.2 \times 10^{0}\right)$ \\
Vibrio sp. S141(p519ngfp) & $\begin{array}{l}1.0 \times 10^{0}\left( \pm 1.2 \times 10^{0}\right) \\
0.9 \times 10^{0}\left( \pm 0.8 \times 10^{0}\right)\end{array}$ \\
Vibrio sp. S141(p519ngfp) & Pseudoalteromonas sp. S91CX (24) & $0.9 \times$ teromonas sp. S91CX (0) \\
\hline
\end{tabular}


Table 4. Experimental inoculation regimes and numbers of GFP cells (mean \pm SD) in biofilms with glutamate on glass

\begin{tabular}{|lcr|}
\hline Strain inoculation at time $0 \mathrm{~h}$ & Strain and time of inoculation (h) & $\begin{array}{c}\mathrm{GFP}^{+} \text {cell numbers } \\
\text { per } 5000 \mu \mathrm{m}^{2} \mathrm{after} 72 \mathrm{~h}\end{array}$ \\
\hline Vibrio sp. S141(p519ngfp) & - & $3.7 \times 10^{1}\left( \pm 1.3 \times 10^{1}\right)$ \\
Vibrio sp. S141(p519ngfp) & $\begin{array}{l}3.3 \times 10^{1}\left( \pm 1.4 \times 10^{1}\right) \\
\text { Vibrio sp. S141(p519ngfp) }\end{array}$ & $\begin{array}{c}\text { Pseudoalteromonas sp. S91(pDSK519) (0) } \\
\text { Pseudoalteromonas sp. S91CX (0) }\end{array}$ \\
\hline
\end{tabular}

\section{DISCUSSION}

Our results indicated that the production of chitinases by Pseudoalteromonas sp. S91(pDSK519) enabled Vibrio sp. S141(p519ngfp) cells to accumulate in numbers in biofilms on squid pen, since no increase in numbers was found when Vibrio sp. S141(p519ngfp) was grown with Pseudoalteromonas sp. S91CX, a chitinase-negative mutant. The simplest explanation for these findings is that Vibrio sp. S141(p519ngfp) was able to use products of squid pen hydrolysis (such as GlcNAc) released into the biofilm by the Pseudoalteromonas sp. S91(pDSK519) chitinases. This was consistent with the results found for dual-species biofilms grown on glass in the presence of glutamate, a soluble carbon source only utilisable by Pseudoalteromonas sp. S91(pDSK519). Under these conditions, Vibrio sp. S141(p519ngfp) cell numbers did not accumulate. In contrast, Psychrobacter sp. SW5\#19, which is unable to use products of chitin degradation, was unable to accumulate in mixed-species biofilms and may have been outcompeted by Pseudoalteromonas sp. S91(pDSK519), since its numbers were significantly decreased in the presence of Pseudoalteromonas sp. S91(pDSK519).

Vetter \& Deming (1999) found that released bacterial extracellular enzymes could remain functional for extended periods of up to $6 \mathrm{~h}$ time and at distances of up to $100 \mu \mathrm{m}$ from enzyme-producing cells. These findings suggest the non-specific activity of hydrolytic enzymes produced by bacterial cells does not assure that the catabolic by-products released following the enzymes' action will be metabolised by the enzymeproducing cells, possibly even being metabolised by cells of different species (Vetter \& Deming 1999). This behaviour can be described as commensal and does not inhibit the fitness of the species being exploited, providing that the resources procured through this process do not become a limiting factor for growth or development of the exploited species. In our study, the ability of Vibrio sp. S141(p519ngfp) to increase in the presence of Pseudoalteromonas sp. S91(pDSK519) in biofilms on squid pen indicated that Vibrio sp. S141(p519ngfp) was able to scavenge or metabolise by-products of squid pen degradation. This would appear to satisfy the definition of a commensal rela- tionship, since Pseudoalteromonas sp. S91SB did not appear to suffer reduced fitness from Vibrio sp. S141 scavenging nutrients. As the majority of bacteria within the marine environment persist as biofilmassociated mixed microbial consortia attached to surfaces (Costerton et al. 1995), interactions of this nature may be common among many species.

Many examples of cross-feeding within mixedspecies biofilms have been published (Moller et al. 1997, Okabe et al. 2005), but few studies have investigated interspecies cross-feeding within biofilms attached to biodegradable substrata. Studies by Baty et al. (2000) found that single-species biofilms of Pseudoalteromonas sp. S91, growing on a solid chitin surface, contained 2 physiologically different populations. It was observed that a relatively small proportion of surface-associated Pseudoalteromonas sp. S91 cells were chitinase positive and primarily responsible for the production of extracellular chitinase enzymes that liberated nutrients from the chitin surface. The second biofilm subpopulation of Pseudoalteromonas sp. S91 did not produce detectable levels of extracellular chitinase. This population also remained associated with the chitin surface but instead took up liberated nutrients and proliferated, producing cells that entered the aqueous phase ready to colonise new surfaces (Baty et al. 2000). This interaction could be viewed one of 2 ways: as social cheating (Velicer 2003) or as a division of labour within a biofilm community resulting in both nutrient liberation and species proliferation (Baty et al. 2000). During our experiment, the Vibrio sp. S141 that scavenged similarly liberated nutrients from the Pseudoalteromonas sp. S91 biofilm may have exploited this intraspecies altruistic behaviour.

A study by Sandoz et al. (2007) found that mutant populations arose, carrying a mutation in the las $R$ gene, within Pseudomonas aeruginosa biofilms from cystic fibrosis patients. These mutant $P$. aeruginosa stopped producing proteases and instead consumed nutrients that were liberated by the proteolytic activity of their wild type neighbours. This is similar to the report by Baty et al. (2000) of the sub-population of Pseudoalteromonas sp. S91 that did not to produce chitinase enzymes, instead consuming the nutrients that were produced by their biofilm neighbours. However, in our study, the ability of Vibrio sp. S141 to accu- 
mulate in numbers within a biofilm of Pseudoalteromonas sp. S91 is an example of an interspecies interaction that resulted in the success of a genetically unrelated population.

It should be noted that, like the subpopulation of proliferating non-enzyme-producing Pseudoalteromonas sp. S91 observed by Baty et al. (2000), Vibrio sp. S141 and Psychrobacter sp. SW5\#19 may have exploited Pseudoalteromonas sp. S91 for growth and detachment, but our experiments were not designed to detect this.

Zhang \& Bishop (2003) found that some biofilmassociated microorganisms were capable of degrading extracellular polymeric substances, produced during biofilm formation, when under conditions of starvation. Additionally, in mixed-species consortia, a species not responsible for extracellular polymeric substance production was seen to benefit from its consumption (Zhang \& Bishop 2003). However, if extracellular polymers produced by Pseudoalteromonas sp. S91(pDSK519) were the source of metabolic support obtained by Vibrio sp. S141(p519ngfp), then polymer production, or the ability of Vibrio sp. S141(p519ngfp) to access this resource, was directly affected when Pseudoalteromonas sp. S91(pDSK519) had lost its ability to produce chitinases or when Pseudoalteromonas sp. S91(pDSK519) was grown on glass. The production of extracellular polymers by biofilm-associated bacteria in mixed-species consortia requires further analysis to determine its role in mediating interspecies interactions.

On squid pen in mixed-species biofilms, Vibrio sp. S141(p519ngfp) cells were localised at the outer edge of the Pseudoalteromonas sp. S91(pDSK519) biofilm (Fig. 1e), whereas in monoculture, Vibrio sp. S141 (p519ngfp) cells were localised directly on the pen surface (Fig. 1c), indicating that Pseudoalteromonas sp. S91(pDSK519) displaced Vibrio sp. S141(p519ngfp), or Vibrio sp. S141(p519ngfp) migrated to a preferred location within the dual-species biofilm. Psychrobacter sp. SW5\#19 cells also attached directly to the pen surface in monoculture (Fig. 1d), and in a dual-species biofilm, remaining Psychrobacter sp. SW5\#19 cells were also localised directly on the squid pen surface. Psychrobacter sp. SW5\#19 is a non-motile marine bacterium, and its inability to be displaced by Pseudoalteromonas sp. S91(pDSK519) or actively migrate within the biofilm may have been a contributing factor as to why it did not persist within dual species.

Few studies have examined changes in biofilm structure or species localisation within the biofilm when challenged with a second species. Fitch et al. (2002) found that Vibrio sp. S141(p519ngfp), provided with a soluble carbon source, is able to displace Psychrobacter sp. SW5H to multiply preferentially on a glass surface in mixed-species biofilms, and Sturman et al. (1994) found that a developed Pseudomonas aeruginosa biofilm attached to an inert pellet was displaced and outcompeted by Klebsiella pneumoniae over a $21 \mathrm{~d}$ period.

Future work employing a non-motile Vibrio sp. S141 mutant (Delpin et al. 2000) will investigate whether motility plays a role in the behavioural interaction between this species and Pseudoalteromonas sp. S91 forming biofilms on squid pen. Furthermore, a nonmotile Pseudoalteromonas sp. S91 mutant may be unable to displace the motile Vibrio sp. S141, which may also change the behavioural interaction between them in mixed-species biofilms.

In conclusion, our results showed that microbial interactions in biofilms growing on biodegradable substrata may be cooperative or competitive and completely differed from those grown on inert substrata. Little information is available in the literature about mixed-species biofilms grown on biodegradable substrata, although this is a major component of natural ecosystems. This represents an important area for further research.

Acknowledgements. K.J.E. was supported by a Flinders University postgraduate scholarship. This work was funded in part by the Flinders University and the Australian Research Council. We are grateful to P. Kolesik for assistance with ImageJ quantification and the expert assistance of the members of the Adelaide Microscopy Unit, J. Terlet, L. Waterhouse, M. Wallwork and P. Self.

\section{LITERATURE CITED}

Abramoff MD, Magelhaes PJ, Ram SJ (2004) Image processing with ImageJ. Biophotonics Int 11:36-42

Albertson NH, Stretton S, Pongpattanakitshote S, Ostling J, Marshall KC, Goodman AE, Kjelleberg S (1996) Construction and use of a new vector/transposon, pLBT:mini Tn10:lac:kan, to identify environmentally responsive genes in a marine bacterium. FEMS Microbiol Lett 140: 287-294

Angles ML (1997) Gene transfer in marine biofilms. PhD dissertation, University of New South Wales, Sydney

Angles ML, Marshall KC, Goodman AE (1993) Plasmid transfer between marine bacteria in the aqueous phase and biofilms in reactor microcosms. Appl Environ Microbiol 59:843-850

Baty AM, Eastburn CC, Diwu Z, Techkarnjanaruk S, Goodman AE, Geesey GG (2000) Differentiation of chitinaseactive and non-chitinase-active subpopulations of a marine bacterium during chitin degradation. Appl Environ Microbiol 66:3566-3573

> Christensen BB, Haagensen JA, Heydorn A, Molin S (2002) Metabolic commensalism and competition in a twospecies microbial consortium. Appl Environ Microbiol 68:2495-2502

Costerton JW, Lewandowski Z, DeBeer D, Caldwell DE, Korber DR, James G (1994) Biofilms, the customized microniche. J Bacteriol 49:2137-2142 
Costerton JW, Lewandowski Z, Caldwell DE, Korber DR, Lappin-Scott HM (1995) Microbial biofilms. Annu Rev Microbiol 49:711-745

> Dalton HM, Poulsen LK, Halasz P, Angles ML, Goodman AE, Marshall KC (1994) Substratum-induced morphological changes in a marine bacterium and their relevance to biofilm structure. J Bacteriol 176:6900-6906

Davies DG, Parsek MR, Pearson JP, Iglewski BH, Costerton JW, Greenberg EP (1998) The involvement of cell-to-cell signals in the development of a bacterial biofilm. Science 280:295-298

Delpin MW, Mclennan AM, Kolesik P, Goodman AE (2000) Comparison of microcolony formation between Vibrio sp. strains S141 and a flagellum-negative mutant developing biofilms on agar and glass substrata. Biofouling 5:183-193

$>$ De Souza ML, Newcombe D, Alvey D, Crowley DE, Hay A, Sadowsky MJ, Wackett LP (1998) Molecular basis of a bacterial consortium: interspecies catabolism of atrazine. Appl Environ Microbiol 64:178-184

Fitch AJ, Kolesik P, Pile AJ, Goodman AE (2002) Plasmid maintenance and localisation of Vibrio sp. S141(p519ngfp) cells within monoculture and mixed-species biofilms. Biofouling 18:275-283

Gooday GW (1990) The ecology of chitin degradation. Adv Microb Ecol 11:387-430

Hale M, Mitchell JG (2001) Functional morphology of diatom frustule microstructures: hydrodynamic control of Brownian particle diffusion and advection. Aquat Microb Ecol 24:287-295

Keen NT, Tamaki S, Kobayashi D, Trollinger D (1988) Improved broad-host-range plasmids for DNA cloning in gram-negative bacteria. Gene 70:191-197

Keyhani NO, Roseman S (1999) Physiological aspects of chitin catabolism in marine bacteria. Biochim Biophys Acta 1473:108-122

Kudo H, Cheng KJ, Costerton JW (1987) Interactions between Treponema bryantii and cellulolytic bacteria in the in vitro degradation of straw cellulose. Can J Microbiol 33: 267-272

Lim NSH, Everuss KJ, Goodman AE, Benkendorff K (2007) Comparison of surface microfouling and bacterial attachment on the egg capsules of two molluscan species representing Cephalopoda and Neogastropoda. Aquat Microb Ecol 47:275-287

Matthysse AG, Stretton S, Dandie C, McClure NC, Goodman AE (1996) Construction of GFP vectors for use in Gramnegative bacteria other than Escherichia coli. FEMS Microbiol Lett 145:87-94

Miller JH (1972) Experiments in molecular genetics. Cold Spring Harbor Laboratory, Cold Spring Harbor, NY

Miller VL, Mekalanos JJ (1988) A novel suicide vector and its use in construction of insertion mutations: osmoregulation of outer membrane proteins and virulence determinants in Vibrio cholera toxR. J Bacteriol 170:2575-2583

Møller S, Korber DR, Wolfaardt GM, Molin S, Caldwell DE (1997) Impact of nutrient composition on a degradative biofilm community. Appl Environ Microbiol 63:2432-2438

Nielsen AT, Tolker-Nielsen T, Barken KB, Molin S (2000) Role of commensal relationships on the spatial structure of a surface-attached microbial consortium. Environ Microbiol 2:59-68

- Okabe S, Kindaichi T, Ito T (2005) Fate of ${ }^{14} \mathrm{C}$-labeled microbial products derived from nitrifying bacteria in autotrophic nitrifying biofilms. Appl Environ Microbiol 71: 3987-3994

Östling J, Goodman AE, Kjelleberg S (1991) Behaviour of InP-1 plasmids and a miniMu transposon in a marine
Vibrio sp. S14: isolation of starvation inducible lac operon fusions. FEMS Microbiol Ecol 86:83-94

O'Sullivan CA, Burrell PC, Clarke WP, Blackall LL (2005) Structure of a cellulose degrading bacterial community during anaerobic digestion. Biotechnol Bioeng 92: 871-878

> O'Toole GA, Kolter K (1998) Flagellar and twitching motility are necessary for Pseudomonas aeruginosa biofilm development. Mol Microbiol 30:295-304

Palmer RJ Jr, Kazmerzak K, Hansen MC, Kolenbrander PE (2001) Mutualism versus independence: strategies of mixed-species oral biofilms in vitro using saliva as the sole nutrient source. Infect Immun 69:5794-5804

> Poulsen LK, Dalton HM, Angles ML, Marshall KC, Molin S, Goodman AE (1997) Simultaneous determination of gene expression and bacterial identity in single cells in defined mixtures of pure cultures. Appl Environ Microbiol 63: 3698-3702

Pratt LA, Kolter R (1998) Genetic analysis of Escherichia coli biofilm formation: roles of flagella, motility, chemotaxis and type I pili. Mol Microbiol 30:285-293

Rao D, Webb JS, Kjelleberg S (2005) Competitive interactions in mixed-species biofilms containing the marine bacterium Pseudoalteromonas tunicata. Appl Environ Microbiol 71:1729-1736

Sandoz KM, Mitzimberg SM, Schuster M (2007) Social cheating in Pseudomonas aeruginosa quorum sensing. Proc Natl Acad Sci USA 104:15876-15881

> Song H, Clarke WP, Blackall LL (2005) Concurrent microscopic observations and activity measurements of cellulose hydrolyzing and methanogenic populations during the batch anaerobic digestion of crystalline cellulose. Biotechnol Bioeng 91:369-378

Sternberg C, Christensen BB, Johansen T, Nielsen AT, Andersen JB, Givsok M, Molin S (1999) Distribution of bacterial growth activity in flow-chamber biofilms. Appl Environ Microbiol 65:4108-4117

> Stretton S, Techkarnjanaruk S, McLennan AM, Goodman AE (1998) Use of green fluorescent protein to tag and investigate gene expression in marine bacteria. Appl Environ Microbiol 64:2554-2559

Sturman P, Jones WL, Characklis WG (1994) Interspecies competition in colonised porous pellets. Water Res 28: 831-839

- Techkarnjanaruk S, Pongpattanakitshote S, Goodman AE (1997) Use of a promoterless lacZ gene insertion to investigate chitinase gene expression in the marine bacterium Pseudoalteromonas sp. strain S9. Appl Environ Microbiol 63:2989-2996

> Velicer GJ (2003) Social strife in the microbial world. Trends Microbiol 11:330-337

- Vetter YA, Deming JW (1999) Growth rates of marine bacterial isolates on particulate organic substrates solubilized by freely released extracellular enzymes. Microb Ecol 37: 86-94

- Wolfaardt GM, Lawrence JR, Robarts RD, Caldwell SJ, Caldwell DE (1994) Multicellular organisation in a degradative biofilm community. Appl Environ Microbiol 60:434-446

Yoshida A, Kuramitsu HK (2002) Streptococcus mutans biofilm formation: utilization of a gtfB promoter-green fluorescent protein (PgtfB:gfp) construct to monitor development. Microbiology 148:3385-3394

Zar JH (1996) Biostatistical analysis, 3rd edn. Prentice-Hall, Upper Saddle River, NJ

Zhang X, Bishop PL (2003) Biodegradability of biofilm extracellular polymeric substances. Chemosphere 50:63-69 